\title{
Potential Impact of Incorporating a Patient-Selected Support Person into mHealth for Depression
}

\author{
James E. Aikens, Ph.D. ${ }^{7}$, Ranak Trivedi, Ph.D. ${ }^{2,3}$, Alicia Heapy, Ph.D. ${ }^{4,5}$, Paul N. Pfeiffer, M.D., M.S. ${ }^{6,7}$, \\ and John D. Piette, MSc, PhD 6,8
}

'Department of Family Medicine, University of Michigan, Ann Arbor, MI, USA; ${ }^{2}$ Center for Innovation to Implementation, VA Palo Alto Health Care System, Palo Alto, CA, USA; ${ }^{3}$ Department of Psychiatry and Behavioral Sciences, Stanford University, Palo Alto, CA, USA; ${ }^{4}$ Pain Research, Informatics, Multimorbidities, and Education (PRIME) Center, VA Connecticut Healthcare System, West Haven, CT, USA; ${ }^{5}$ Department of Psychiatry, Yale University School of Medicine, New Haven, CT, USA; ${ }^{6}$ Center for Clinical Management Research, VA Ann Arbor Health System, Ann Arbor, MI, USA; ${ }^{7}$ Department of Psychiatry, University of Michigan Medical School, Ann Arbor, MI, USA; ${ }^{8}$ Department of Health Behavior and Health Education, School of Public Health, University of Michigan, Ann Arbor, MI, USA.

BACKGROUND: Although telephone care management improves depression outcomes, its implementation as a standalone strategy is often not feasible in resourceconstrained settings. Moreover, little research has examined the potential role of self-management support from patients' trusted confidants.

OBJECTIVE: To investigate the potential benefits of integrating a patient-selected support person into automated mobile health (mHealth) for depression.

DESIGN: Patient preference trial.

PARTICIPANTS: Depressed primary care patients who were at risk for antidepressant nonadherence (i.e., Morisky Medication Adherence Scale total score $>1$ ).

INTERVENTION: Patients received weekly interactive voice response (IVR) telephone calls for depression that included self-management guidance. They could opt to designate a lay support person from outside their home to receive guidance on supporting their self-management. Patients' clinicians were automatically notified of urgent patient issues.

MAIN MEASURES: Each week over a period of 6 months, we used IVR calls to monitor depression with the Patient Health Questionnaire-9 (PHQ-9; with total<5 classified as remission), adherence (single item reflecting perfect adherence over the past week), and functional impairment (any bed days due to mental health).

KEY RESULTS: Of 221 at-risk patients, $61 \%$ participated with a support person. Analyses were adjusted for race, medical comorbidity, and baseline levels of symptom severity and adherence. Significant interaction effects indicated that during the initial phase of the program, only patients who participated with a support person improved significantly in their likelihood of either adhering to antidepressant medication (AOR=1.31, 95\% CI: 1.16-1.47, $p<0.001$ ) or achieving remission of depression symptoms (AOR=1.24, 95\% CI: 1.14-1.34, $p<0.001)$. These benefits were maintained throughout the 6 -month observation period.

CONCLUSIONS: Incorporating the "human factor" of a patient-selected support person into automated mHealth

Received July 28, 2014

Revised November 18, 2014

Accepted January 21, 2014

Published online February 10, 2015 for depression self-management may yield sustained improvements in antidepressant adherence and depression symptom remission. However, this needs to be confirmed in a subsequent randomized controlled trial.

KEY WORDS: depression; mHealth; self-management; caregiving; social support.

J Gen Intern Med 30(6):797-803

DOI: $10.1007 / \mathrm{s} 11606-015-3208-7$

(C) Society of General Internal Medicine 2015

\section{INTRODUCTION}

Depression is a prevalent and potentially debilitating condition that is projected to become the second most burdensome disease worldwide by $2030 .^{1}$ Antidepressant medication effectively reduces symptom severity and recurrence risk, but nonadherence is common. ${ }^{2,3}$ Although telephone care management is an evidence-based and cost-effective strategy for improving antidepressant medication adherence, ${ }^{4}$ its implementation is often not feasible in resource-constrained settings due to high numbers of undertreated patients and limited numbers of trained providers. ${ }^{5}$

Mobile health (mHealth) services such as interactive voice response (IVR) telephone calls, smartphone applications, and text messaging may help to address these problems. In IVR calls, patients respond to prerecorded queries using either their telephone keypad or voice recognition technology. We have designed and implemented an mHealth IVR system named "CarePartners," 6 in which patients undergo weekly automated IVR telephone assessments and receive messages regarding their medication adherence and symptom control, and their clinicians are automatically notified of urgent problems such as acute worsening of depression symptoms.

Because many patients attribute their depression to insufficient social support, ${ }^{7}$ and those with high-quality support have better outcomes, ${ }^{8,9}$ it may also be beneficial to intensify the self-management support that patients receive from trusted confidants. While their significant others often provide invaluable assistance, these helpers are at high risk for psychological distress. ${ }^{10}$ Friends or relatives from outside the household may 
be willing to assist, but they usually lack effective tools for systematically monitoring patients' moods and encouraging effective self-management. To address these issues, a program was designed that would allow patients to choose an informal support person who automatically receives structured updates about their depression status, along with problem-specific guidance on supporting their self-management.

To our knowledge, the CarePartners program is the only patient-centered automated mHealth program that integrates a member of the patient's social support network. We have established that the program has a high rate of engagement, yields valid and reliable clinical information, generates a manageable number of clinician notifications, and responds appropriately to urgent situations when implemented for depression $^{6}$ as well as for four other chronic conditions. ${ }^{11}$ However, we are unaware of any other telephonic programs incorporating a support person. Therefore, this study tests the hypothesis that patients who choose to include a support person in their mHealth program show better improvement in adherence, symptoms, and function than those who participate in the same program without a support person.

\section{METHODS}

\section{Patient Eligibility and Recruitment}

Patients. Patients were enrolled between March 2010 and January 2012 from 13 primary care practices. To be eligible, patients had to have had two primary care visits within the previous two years and at least one within the previous 13 months, and either (a) an active depression diagnosis or (b) an antidepressant prescription, together with a billing diagnosis of depression. We excluded patients with a diagnosis of psychotic disorder, bipolar disorder, or dementia. We mailed an introductory letter to potentially eligible patients and then solicited their participation by telephone. Participants provided written informed consent. Given our focus upon medication adherence, we selected for data analysis only patients who were at risk for nonadherence, as indicated by the well-validated Morisky Medication Adherence Scale (MMAS). ${ }^{12}$ Higher MMAS scores reflect poorer adherence, and we used a stringent cutoff of $>1$ to identify baseline risk for nonadherence. The protocol was approved by our institutional review board.

Support Person. At enrollment, patients were offered the option of participating together with a supportive family member or friend. We used the Norbeck Social Support Questionnaire (NSSQ) ${ }^{13}$ to facilitate their identification of the most appropriate support person. Support persons were required to be at least 18 years old, have no history of psychosis or cognitive impairment, and agree to receive electronic reports based on information reported during the patient's IVR calls.

\section{Baseline Assessment}

Following the informed consent process, patients provided information on their sociodemographic and clinical characteristics. At baseline only, we used the eight-item version of the Patient Health Questionnaire (PHQ-8) ${ }^{14}$ to evaluate depression symptom severity. It is identical to the PHQ-9, but without the suicidality item. As noted above, we used the MMAS to assess baseline nonadherence to antidepressants. Health literacy was measured with an abbreviated screen based upon the Test of Functional Health Literacy in Adults, which provides a binary indicator of inadequate health literacy. ${ }^{15}$ We estimated number of comorbid chronic conditions based on the self-reported presence of hypertension, cardiovascular disease, hyperlipidemia, stroke, arthritis, chronic lung disease, and low back pain.

\section{Intervention Program}

General Program Structure and Processes. The program provided (a) the patient with IVR monitoring of depression symptoms and medication adherence, including tailored information about self-care and when to seek health services; (b) the patient's clinical team with actionable feedback about IVR-reported problems; and (c) the support person with problem-tailored guidance on providing self-management support. Patients were scheduled to receive weekly IVR calls. Each week that an IVR call was scheduled (i.e., "call-week"), the system made up to nine call attempts at patient-selected day/time combinations. Call contents were developed by a multidisciplinary team, and calls typically lasted 5-7 min. The program was provided to all patients for at least six months.

IVR-Based Assessments. Patients' symptoms of depression were assessed during each call using the PHQ- $9^{16}$ (as opposed to the PHQ- $8,{ }^{14}$ which was used only at baseline). We defined remission (a primary outcome) as PHQ-9 total of $<5$. Acute clinical worsening was defined as a PHQ-9 increase $\geq 7$ points since the preceding assessment. If patients reported possible thoughts of self-harm or of "being better off dead," they were then asked follow-up questions to assess their safety. Adherence to antidepressant medication was assessed during IVR calls by asking: "Many people have difficulty taking their medicine exactly as prescribed by their doctor. How often would you say you took your depression medicine exactly as prescribed this past week?" (on a scale ranging from 1 ("always") to 4 ("rarely or never"). We stringently dichotomized adherence as perfect versus less than perfect (another primary outcome), which tends to correct for inflated adherence self-reporting. ${ }^{2,17}$ The IVR system also assessed functional impairment (the third primary outcome) by asking whether the patient had stayed in bed for any entire day during the past week due to mental health problems. 
Patient Self-Management Messages. For each major area assessed during the IVR calls, patients heard either a positive acknowledgement message when they reported doing well, or a problem-specific self-management support message when they reported a difficulty. Patients reporting suicidal ideation were advised to discuss this with their physician, and given the number of a 24-hour suicide hotline. Those meeting additional criteria for suicidal risk were also given information on emergency assistance and offered immediate transfer to a suicide hotline.

Clinician Alerts. Clinician alerts were triggered if: (a) the IVR data indicated elevated suicide risk, (b) the PHQ-9 score increased by either $\geq 7$ or from $<15$ to $\geq 15$ since the preceding assessment, (c) the patient reported either "rarely or never" taking their antidepressant as prescribed, or (d) the patient reported reducing or discontinuing their antidepressant without medical supervision.

Support Persons' Reports. Patients' designated support persons were emailed a report after each completed call that summarized the patient's symptoms, impairment level, adherence, and miscellaneous issues. This included detailed suggestions on strategies for supporting the patient's selfmanagement. Further details on the intervention program are available from the authors upon request.

\section{Data Analysis}

We analyzed the data using STATA $11.2{ }^{18}$ Initial descriptive analyses were used to characterize patient participants overall and by groups defined by whether or not the patient participated with a support person. We evaluated group differences in baseline characteristics using Student's t tests for continuous variables and chi-squared tests for categorical variables. Next, the primary analyses were conducted at the level of callweek within patients and included all available data (i.e., no data were imputed). We divided the study timeline into two phases for analysis: acute (weeks 1-6) and maintenance (weeks 7-26). Our primary analyses used multivariable logistic regression to evaluate group differences in the odds of adhering to antidepressant medication, achieving symptom remission (i.e., PHQ-9<5), and having at least one bed day due to depression. We adjusted all models for baseline symptom severity (PHQ-8) and demographic covariates selected through preliminary analysis, and we further adjusted the adherence model for baseline adherence (MMAS). The primary effect of interest was the group $\mathrm{X}$ week interaction term, which we followed up within groups. We plotted significant effects across weeks, with locally weighted scatterplot smoothing (LOWESS) ${ }^{19}$ to facilitate visual interpretation. We used a $p$ criterion of $<0.05$ to judge statistical significance after adjusting standard errors for clustering of call-weeks within patients, except for preliminary analyses to identify confounders, for which we used a criterion of $p<0.10$.

\section{RESULTS}

\section{Patient Characteristics}

Of the 1,330 patients contacted, we qualified and enrolled 387, of which 221 (57\%) met criteria for being at risk for nonadherence and therefore comprised the analytic sample (see Table 1). The typical participant was a married white woman with at least some postsecondary education. Participants were 50 years of age on average, with $19 \% \geq 60$ years old and approximately half with $\geq 3$ chronic comorbid conditions. Baseline PHQ8 scores indicated "moderate" severity on average, with about one-fourth of patients meeting criteria for either symptom remission or at least "moderately severe" symptoms, respectively. Sixty-one percent of patients enrolled with a support person (SP group, $n=135$ ), while the remainder enrolled alone (control group, $n=86$ ).

Table 1 Patient Sample Characteristics

\begin{tabular}{|c|c|c|c|c|}
\hline \multirow[b]{2}{*}{ Baseline variable } & \multicolumn{4}{|c|}{ Percentage or mean \pm SD } \\
\hline & $\begin{array}{l}\text { Pooled sample } \\
(n=221)\end{array}$ & $\begin{array}{l}\text { Group } \\
\text { SP } \\
(n=135)\end{array}$ & $\begin{array}{l}\text { Control } \\
(n=86)\end{array}$ & $p$ \\
\hline Age & $51.4 \pm 12.7$ & $50.7 \pm 11.9$ & $51.9 \pm 13.1$ & 0.477 \\
\hline Female gender & 78.6 & 74.8 & 84.7 & 0.084 \\
\hline Minority ethnicity & 9.5 & 6.7 & 14.0 & 0.078 \\
\hline No post-secondary education & 20.8 & 21.5 & 19.8 & 0.760 \\
\hline Annual income $<\$ 30,000$ & 31.8 & 35.3 & 26.2 & 0.160 \\
\hline Married & 56.1 & 60.0 & 50.0 & 0.145 \\
\hline Number of comorbid conditions $\mathrm{s}^{\mathrm{a}}$ & $3.5 \pm 2.4$ & $3.7 \pm 2.5$ & $3.2 \pm 2.2$ & 0.087 \\
\hline Low functional health literacy ${ }^{\mathrm{b}}$ & 16.8 & 17.8 & 15.1 & 0.591 \\
\hline Adherence (MMAS) ${ }^{\mathrm{c}}$ & $1.6 \pm 0.8$ & $1.6 \pm 0.8$ & $1.6 \pm 0.7$ & 0.575 \\
\hline Depression symptom severity (PHQ-8) ${ }^{\mathrm{d}}$ & $10.2 \pm 6.2$ & $10.7 \pm 6.0$ & $9.3 \pm 6.3$ & 0.091 \\
\hline Symptom remission $\left(\mathrm{PHQ}-8^{\mathrm{a}}<5\right)$ & 20.7 & 17.3 & 26.2 & 0.117 \\
\hline
\end{tabular}

${ }^{a}$ Hypertension, cardiovascular disease, hyperlipidemia, stroke, arthritis, chronic lung disease (asthma or COPD), or low back pain

${ }^{b}$ As measured by the Test of Functional Health Literacy for Adults, brief version

${ }^{c}$ Morisky Medication Adherence Scale, for which higher scores indicate lower adherence

${ }^{d}$ Patient Health Questionnaire-8, for which higher scores indicate more severe symptoms. 


\section{Associations Between Baseline Features and Participating with a Support Person}

The SP and control groups did not significantly differ in age $(p=0.477)$, having annual household income $<\$ 30,000$ $(p=0.160)$, having no education beyond high school $(p=0.760)$, being married $(p=0.145)$, having low functional health literacy $(p=0.591)$, or adhering to medication at baseline $(p=0.575)$. Likewise, group designation was not significantly associated with living alone $(p=0.873)$, household size ( $p=0.385)$, number of immediate family members $(p=0.644)$, or having children at home $(p=0.735)$. However, patients in the SP group were less likely to be female $(\mathrm{OR}=0.54,95 \% \mathrm{CI}$ : $0.27-1.09, p=0.084)$ or an ethnic minority ( $\mathrm{OR}=0.44,95 \% \mathrm{CI}$ : $0.18-1.10, p=0.078)$. They also may have had more comorbid medical conditions $(\mathrm{OR}=1.10,95 \% \mathrm{CI}$ : $0.96-1.24, p=0.087)$ and higher baseline depression symptom severity $(\mathrm{OR}=1.04$, 95 \% CI: $0.99-1.09, p=0.091)$. When these latter four variables were tested simultaneously, the $p$ value for gender increased to 0.356 , whereas there was little appreciable change in the $p$ values for the other three variables. Therefore, we selected minority ethnicity, comorbidity, and symptom severity as control covariates.

\section{Descriptive Data on IVR Calls}

Patients participated for a median of 25 of a possible 26 weeks (interquartile range: 22-26), yielding 5,739 patient-weeks of follow-up. Attrition (defined as actively withdrawing from the study or completing $<50 \%$ of scheduled IVR calls) was seen in $11 \%$ and was unrelated to baseline symptoms ( $p=0.675)$, study group $(p=0.381)$, or sociodemographic characteristics (all $p$ values $>0.05$ ). Patients completed IVR calls in $72 \%$ of call-weeks during which one was attempted, yielding 3,023 completed calls for analysis: 1,464 from the acute phase and 1,559 from the maintenance phase.

\section{Associations Between Group and IVR-Reported Primary Outcomes}

We conducted logistic regression analyses to evaluate whether study group (SP vs. control) was associated with the three primary outcomes generated via IVR calls. All models were run separately for the acute and maintenance phases. Preliminary analyses indicated no group-related differences in call completion for either unadjusted or adjusted models (both $p$ values $\geq 0.209$ ).

Antidepressant Adherence. Results for the acute phase are shown in Table 2. We adjusted the model for baseline severity (PHQ-8) and baseline nonadherence (MMAS). Adherence was more likely among patients with $\geq 3$ comorbid medical conditions (adjusted odds ratio $[\mathrm{AOR}]=1.59,95 \% \mathrm{CI}$ : $1.01-$ 2.48, $p=0.042)$, lower MMAS scores $(\mathrm{AOR}=0.71,95 \% \mathrm{CI}$ : $0.55-0.91, p=0.007)$, and lower baseline PHQ-8 scores (AOR $=0.95,95 \%$ CI: $0.91-0.99, p=0.009)$.

The group $\mathrm{X}$ week interaction was significant $(\mathrm{AOR}=1.18,95 \% \mathrm{CI}: 1.01-1.39, p=0.044)$, indicating that, relative to controls, patients in the SP group became increasingly more likely over time to report adherence. Follow-up analysis indicated that the odds of adherence increased $31 \%$ per week in the SP group $(\mathrm{AOR}=1.31,95 \% \mathrm{CI}: 1.16-1.47, p<0.001)$, but only

Table 2 Logistic Regression Models Predicting Weekly Outcomes During the Acute Phase of the Intervention Program (n=1,464 calls with 221 patients)

\begin{tabular}{|c|c|c|c|}
\hline Variable & AOR & $95 \%$ CI & $p$ value \\
\hline \multicolumn{4}{|l|}{ Model 1, predicting antidepressant adherence } \\
\hline Minority & 1.05 & $0.48-2.29$ & 0.906 \\
\hline$\geq 3$ Comorbid conditions & 1.59 & $1.01-2.48$ & 0.042 \\
\hline Baseline depression symptom severity (PHQ-8) & 0.95 & $0.91-0.99$ & 0.009 \\
\hline Baseline nonadherence (MMAS) & 0.71 & $0.55-0.91$ & 0.007 \\
\hline Group $^{\mathrm{a}}$ & 0.94 & $0.53-1.66$ & 0.821 \\
\hline Week & 1.11 & $0.99-1.24$ & 0.082 \\
\hline Group X week & 1.18 & $1.01-1.39$ & 0.044 \\
\hline \multicolumn{4}{|l|}{ Model 2, predicting depression remission ${ }^{\mathrm{b}}$} \\
\hline Minority & 1.70 & $0.90-3.18$ & 0.100 \\
\hline$\geq 3$ Comorbid conditions & 0.95 & $0.61-1.48$ & 0.812 \\
\hline Baseline depression symptom severity (PHQ-8) & 0.87 & $0.84-0.91$ & $<0.001$ \\
\hline Group $^{\mathrm{a}}$ & 1.16 & $0.72-1.89$ & 0.543 \\
\hline Week & 1.08 & $1.00-1.18$ & 0.065 \\
\hline Group X week & 1.14 & $1.02-1.28$ & 0.027 \\
\hline \multicolumn{4}{|l|}{ Model 3 , predicting any bed days due to depression } \\
\hline Minority & 0.86 & $0.40-1.87$ & 0.712 \\
\hline$\geq 3$ Comorbid conditions & 1.17 & $0.69-2.00$ & 0.550 \\
\hline Baseline depression symptom severity (PHQ-8) & 1.10 & $1.06-1.16$ & $<0.001$ \\
\hline Group $^{\mathrm{a}}$ & 0.70 & $0.38-1.28$ & 0.248 \\
\hline Week & 0.89 & $0.78-1.01$ & 0.070 \\
\hline Group X week & 0.96 & $0.81-1.14$ & 0.628 \\
\hline
\end{tabular}

AOR Adjusted odds ratio

MMAS Morisky Medication Adherence Scale

PHQ-8 Patient Health Questionnaire, 8-item version

${ }^{a}$ Control and $C P$ groups were coded as 0 and 1 , respectively

${ }^{b}$ Based upon the weekly PHQ-9 administered by IVR 
$11 \%$ in the control group $(\mathrm{AOR}=1.11,95 \% \mathrm{CI}: 0.99$ $1.24, p=0.070$ ).

In contrast, analysis of maintenance-phase data indicated that time neither had a significant main effect $(p=0.827)$ nor did it interact with group $(p=0.524)$. This pattern is apparent in Fig. 1, which depicts an acute-phase benefit favoring the SP group, and which remained stable during the maintenance phase.

Depression Remission. As can be seen in Table 2 (Model 2 ), the adjusted odds of achieving remission during the acute phase were lower among patients who had more severe symptoms at baseline (AOR $=0.87,95 \% \mathrm{CI}$ : 0.84-0.91, $p<0.001)$. Moreover, group interacted with time $(\mathrm{AOR}=1.14,95 \% \mathrm{CI}: 1.02-1.28, p=0.07)$, such that patients in the SP group were more likely than controls to achieve remission For the SP group, the odds of remission increased $24 \%$ each week $(\mathrm{AOR}=1.24,95 \% \mathrm{CI}: 1.14-1.34, p<0.001)$, but for the control group only $8 \%$ per week $(\mathrm{AOR}=1.08,95 \% \mathrm{CI}$ : $0.999-1.181, p=0.063)$. During the maintenance phase, neither week nor group $\mathrm{X}$ week had a significant effect ( $p=0.938$ and 0.670 , respectively). Likewise, the weekly plot of the effects by group (see Fig. 2) shows stronger initial improvement in the SP group, followed by good long-term maintenance.

Bed Days due to Depression. The only significant predictor of bed days during the acute phase was baseline symptom severity. Specifically, the odds of having any bed days increased along with baseline severity $(\mathrm{AOR}=1.10,95 \% \mathrm{CI}$ : $1.06-1.16, p<0.001)$. Although there was a possible trend for bed days to become less likely over time $\mathrm{k}(p=0.070)$, this did not differ by group $(p=0.628)$. Similar to the first two outcome variables, the effects of time and the group $\mathrm{X}$ time term were not significant during the maintenance phase $(p=0.415$ and 0.323 , respectively).

\section{DISCUSSION}

Although we cannot draw definitive causal conclusions given the non-experimental study design, the results agreed with our prediction that patients who chose to integrate a support person into an mHealth program for management of depression would show greater improvement in adherence and depression symptoms than those who participated in the same program without a support person. It is important to emphasize that these benefits were observed over and above those of a fairly active control condition.

The program consisted of automated weekly IVR calls with clinician feedback. Patients could opt to select a supportive family member or friend who would be automatically sent structured summaries of their IVR calls, along with suggestions for how they could help support patients' self-management. Specifically, for patients who chose to involve a support person, the odds of antidepressant medication adherence during the program's acute phase increased $31 \%$ per week (versus $11 \%$ for controls), and the odds of depression symptom remission increased $24 \%$ per week (versus $8 \%$ for controls). Both of these relative advantages were sustained for up to six months. However, the results did not support our hypothesis that the same benefit would occur for functional impairment.

Because adherence to antidepressant medication improves depression outcomes ${ }^{20,21}$ increased adherence could be one pathway by which extra support may enhance effectiveness. However, symptoms of depression typically require at least 3 to 4 weeks to respond to pharmacotherapy. If this were the only operative mechanism, improvement in symptoms would probably not occur synchronously with — but rather would lag behind - improved adherence (see Fig. 1a and b).

We therefore believe that regular supportive contact from a trusted and helpful confidant played a role in relieving symptoms independent of medication, perhaps by directly ameliorating the interpersonal isolation and loneliness often experienced by depressed individuals. ${ }^{7-9}$ This interpersonal element

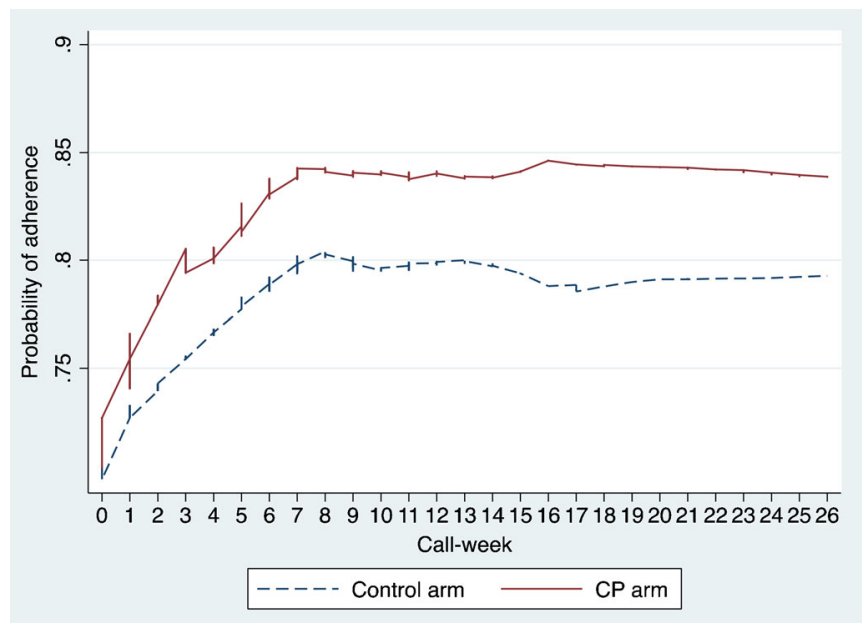

Fig. 1 Probability of adherence by group and call-week 


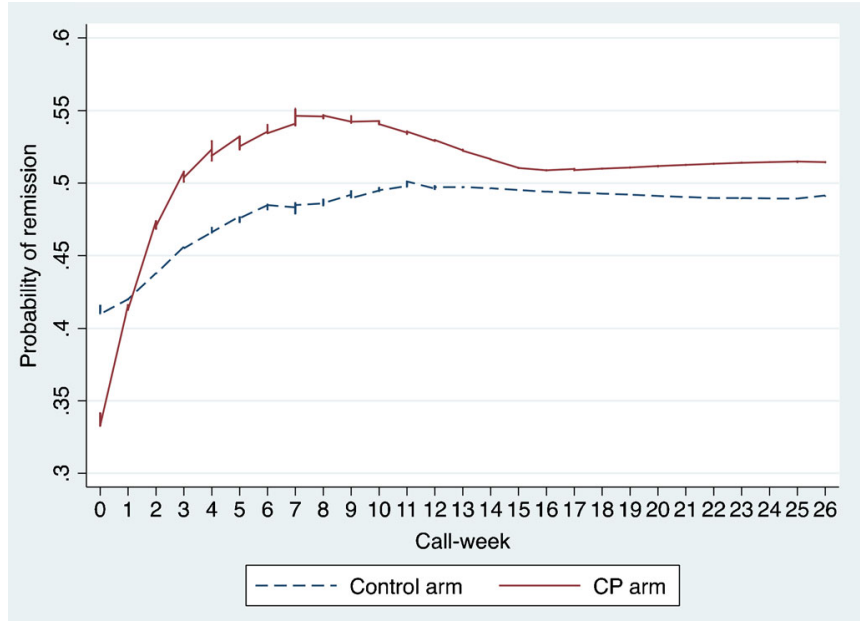

Fig. 2 Probability of remission by group and call-week.

may have also helped to offset the potentially impersonal and/ or repetitive nature of automated IVR. Although bed days due to depression were not affected in either study group, this is a relatively severe form of impairment that occurred during only about $15 \%$ of call-weeks. For patients whose symptoms fall within the mild to moderate range, subtler forms of impairment (e.g., presenteeism, diminished role performance, reduced social functioning) may be more responsive to intensified self-management support. Notwithstanding, functional impairment remains a vitally important behavior to target, given that rigorous trials of behavioral activation indicate that even a few months of better functioning significantly improves the long-term course of depression. ${ }^{22}$

The primary limitation of this study is its nonexperimental design, specifically that study group assignment was determined by patient preference rather than randomly. As such, we cannot draw definitive conclusions whether intensifying self-management support truly improves mHealth outcomes. A number of unmeasured factors might explain patients' decisions to participate with a support person, including the availability of a willing and capable individual, patient preference for connectedness versus privacy, the need for independence, perceived stigma, social anxiety, and social skills. Our data suggest that it was the more depressed and medically complicated patients who tended to choose this option, which implies that support may appeal to the most vulnerable patients who need it the most. Given that patients who participated with a support person had more severe depression and more comorbid medical conditions prior to program initiation, their relative benefits in adherence and remission are even more striking. While from a traditional trialist perspective, preference designs such as the present one may compromise internal validity, they often enhance ecological realism and external validity due to their closer resemblance to real world clinical care. Moreover, potential confounders that arose from nonrandom assignment may have been mitigated by our statistical adjustment for minority status, medical comorbidity, baseline adherence, and baseline symptom severity.

Another study limitation was our reliance upon selfreporting, although our stringent adherence threshold and brief reporting interval likely offset social desirability and recall biases. $^{2,17}$ In a related issue, disclosure of problems might be inhibited if patients are uncomfortable with the automated interface or believe that denying problems will help abbreviate their IVR calls. Despite the fact that IVR assessment is a wellvalidated method for measuring depression symptoms, ${ }^{23}$ follow-up studies should include confirmatory data sources such as electronic medication event monitoring, medical records, and corroboration from significant others. We did not assess adherence to non-pharmacologic therapies (e.g., psychotherapy), nor did we analyze differential effects by specific antidepressant. Although we measured several outcomes from the perspective of patients' support persons, these results will be presented elsewhere. Finally, while the sample was heterogeneous in terms of age, symptom severity, and clinical setting, generalizability may be limited by the preponderance of white women with a post-secondary level of education.

In conclusion, our encouraging preliminary findings suggest that depressed patients' existing social supports are an important but underutilized resource that can be integrated into mHealth interventions to improve antidepressant adherence and mood outcomes. These support persons are presumably motivated to help, but lack the structure and tools needed to do so. If their involvement is amply structured and sufficiently long-term in nature, it may benefit patients clinically while also relieving potentially overextended clinicians and in-home support persons. Our conclusions require confirmation by future research that includes randomized group assignment and multiple data sources. Given the chronic shortages in health care financing and available care management personnel, incorporating patient-designated support persons into automated mHealth programs may help to 
close the gap between patient needs and the very real limitations inherent in many resource-constrained health care settings.

Acknowledgments: We appreciate the statistical consultation that was generously contributed by Ananda Sen, Ph.D., and the editorial assistance provided by Katie Grode, both at the University of Michigan. Nicole Marinec and Dana Striplin provided essential input into the design and technical management of the CarePartners program. JEA and JDP were supported by NIH grant 1R01MH096699, and JDP received additional support from NIH grant P3ODK092926. JDP is a VA Senior Research Career Scientist, RT is a VA Career Development awardee (CDA-09-206), and AH is a VA Health Services Research \& Development awardee (IIR 09-058). The views expressed in this manuscript are those of the authors and do not necessarily reflect the views of the Department of Veterans Affairs. Blue Cross Blue Shield of Michigan provided financial support for program development, and the University of Michigan Health System provided support for program implementation. None of the authors have any financial or personal relationships to disclose with organizations that could be perceived as influencing this research, and there has been no significant financial support for this work that could have influenced its outcome.

Conflict of Interest: The authors each declare no conflicts of interest.

Corresponding Author: James E. Aikens, Ph.D.; Department of Family MedicineUniversity of Michigan, 1018 Fuller Street, Ann Arbor, MI 48104-1213, USA (e-mail: aikensj@umich.edu).

\section{REFERENCES}

1. Mathers C, Fat D, Boerma J. The global burden of disease : 2004 update. Geneva: World Health Organization; 2008.

2. Stirratt M DJ, Crane H, Simoni J, Czajkowski S, Hillard M, Kahana S, Aikens JE, Hunter C, Velligan D, Huntley C, Rand C, Ogedegbe G, Nilsen W. Self-report measures of medication adherence behavior: Recommendations on optimal use. Manuscript under review 2014.

3. Aikens JE, Nease JDE, Nau DP, Klinkman MS, Schwenk TL. Adherence to maintenance-phase antidepressant medication as a function of patient beliefs about medication. Annals of Family Medicine. 2005;3:23-30.

4. Chong W, Aslani P, Chen T. Effectiveness of interventions to improve antidepressant medication adherence: A systematic review. International Journal of Clinical Practice. 2011;65:954-75.

5. Williams JWJ, Rost K, Dietrich AJ, Ciotti MC, Zyzanski SJ, Cornell J. Primary care physicians' approach to depressive disorders. Effects of physician specialty and practice structure. Archives of Family Medicine. 1999;8:58-67.

6. Piette JD, Aikens JE, Trivedi R, Parrish D, Standiford C, Marinec N, Striplin D, Bernstein SJ. Depression self-management assistance using automated telephonic assessments and social support. American Journal of Managed Care. 2013;19:892-900.
7. Strine T, Kroenke K, Dhingra S, Balluz LS, Gonzalez O, Berry JT, Mokdad AH. The associations between depression, health-related quality of life, social support, life satisfaction, and disability in communitydwelling US adults. Jourmal of Nervous and Mental Disease. 2009; 197:61-4.

8. Gladstone GL, Parker GB, Malhi GS, Wilhelm KA. Feeling unsupported? An investigation of depressed patients' perceptions. Journal of Affective Disorders. 2007; 103:147-54.

9. Burcusa SL, Iacono WG. Risk for recurrence in depression. Clinical Psychology Review. 2007;27:959-85.

10. Angermeyer MC, Bull N, Bernert S, Dietrich S, Kopf A. Burnout of caregivers: a comparison between partners of psychiatric patients and nurses. Archives of Psychiatric Nursing. 2006;20:158-65.

11. Piette JD, Rosland AM, Marinec NS, Striplin D, Bernstein SJ, Silveira MJ. Engagement with automated patient monitoring and self-management support calls: Experience with a thousand chronically-ill patients. Medical Care. 2013;51:216.

12. Morisky DE, Green LW, Levine DM. Concurrent and predictive validity of a self-reported measure of medication adherence. Medical Care. 1986;24:67-74.

13. Attkisson CC, Zwick R. The client satisfaction questionnaire. Psychometric properties and correlations with service utilization and psychotherapy outcome. Evaluation \& Program Planning. 1982:5:233-7.

14. Kroenke K, Strine TW, Spitzer RL, Williams JB, Berry JT, Mokdad AH. The PHQ- 8 as a measure of current depression in the general population. Journal of Affective Disorders. 2009;114:163-73.

15. Chew LD, Bradley KA, Boyko EJ. Brief questions to identify patients with inadequate health literacy. Family Medicine. 2004;36:588-94.

16. Kroenke K, Spitzer RL. The PHQ-9: A new depression diagnostic and severity measure. Psychiatric Annals. 2002;32:1-7.

17. Garber MC, Nau DP, Erickson SR, Aikens JE, Lawrence JB. The concordance of self-report with other measures of medication adherence: a summary of the literature. Medical care. 2004;42:649-52.

18. StataCorp. Stata Statistical Software: Release 11. College Station, TX: StataCorp LP 2009.

19. Cleveland WS. Robust locally weighted regression and smoothing scatterplots. Journal of the American Statistical Association. 1979;74:829-36.

20. Aikens JE, Nease DE Jr, Nau DP, Klinkman MS, Schwenk TL. Adherence to maintenance-phase antidepressant medication as a function of patient beliefs about medication. Annals of Family Medicine. 2005;3:23-30. Ann Fam Med. 2005; 3(6):558.

21. Katon WJRJ, Heckbert SR, Lin EHB, Ciechanowski P, Ludman E, Young B, Von Korff $\mathbf{M}$. The relationship between changes in depression symptoms and changes in health risk behaviors in patients with diabetes. International Journal of Geriatric Psychiatry. 2010;25:466-75.

22. Dimidjian S, Hollon SD, Dobson KS, et al. Randomized trial of behavioral activation, cognitive therapy, and antidepressant medication in the acute treatment of adults with major depression. Journal of Consulting and Clinical Psychology. 2006;74:658.

23. Moore HK, Mundt JC, Modell JG, et al. An examination of 26,168 Hamilton Depression Rating Scale scores administered via interactive voice response across 17 randomized clinical trials. Journal of Clinical Psychopharmacology. 2006;26:321-4. 